\title{
RESEARCH
}

Open Access

\section{Client satisfaction to methadone maintenance treatment program in Myanmar}

\author{
Sun Tun ${ }^{1,2^{*}}$ (D, Balasingam Vicknasingam², Darshan Singh ${ }^{2}$ and Nyunt Wai $^{1}$
}

\begin{abstract}
Background: To tackle the long-standing opioid misuse problem, Myanmar introduced the methadone maintenance treatment (MMT) program in 2006, starting with 260 clients. Since then, the program has been expanded across different geographical sites in the country. This study was done in 2017 to explore the treatment satisfaction of the clients towards the MMT program.

Method: A total of 210 clients with a minimum of six-month treatment history enrolled in five MMT program sites across Myanmar were recruited through stratified random sampling. Administering the Verona Service Satisfaction Scale for Methadone-Treatment (VSSS-MT), this study assessed the satisfactory responses under three categories viz., 1) clinic staff professional skills; 2) basic drug intervention; 3) specific intervention (individual rehabilitation and psychotherapy).
\end{abstract}

Results: The majority $(89 \%, n=186)$ of the respondents were satisfied with the current MMT program. Specifically, 89.5\% ( $n=187)$ were satisfied with the clinic staff professional skills category, $91.9 \%(n=192)$ with the basic program intervention and $74.6 \%(n=156)$ with specific interventions. Among the respondents, treatment satisfaction with the MMT program was higher $(p<0.05)$ in those (i) with a higher quality of life score in physical, psychological, social and environmental categories, ii) who were satisfied with their current marital and leisure status, and those iii) who consumed alcohol. Results from stepwise binary logistic regression showed alcohol consumption and physical health status had a significant association with MMT treatment satisfaction.

Conclusion: Treatment satisfaction of the clients, in general is high. However, the lower percentage of satisfied clients (74.6\%) for the specific interventions category compared with 89.5 and $91.9 \%$ respectively for staff and basic drug management categories highlights the need for improvement in this category for overall enhancement of the MMT program.

Keywords: Methadone, Verona service satisfaction scale for methadone treatment - VSSS-MT, Treatment satisfaction, Opiate, Myanmar

\footnotetext{
* Correspondence: suntun1@gmail.com

${ }^{1}$ Myanmar Medical Association, Yangon, Myanmar

${ }^{2}$ Centre for Drug Research, Universiti Sains Malaysia, Penang, Malaysia
}

(c) The Author(s). 2022, corrected publication 2022. Open Access This article is licensed under a Creative Commons Attribution 4.0 International License, which permits use, sharing, adaptation, distribution and reproduction in any medium or format, as long as you give appropriate credit to the original author(s) and the source, provide a link to the Creative Commons licence, and indicate if changes were made. The images or other third party material in this article are included in the article's Creative Commons licence, unless indicated otherwise in a credit line to the material. If material is not included in the article's Creative Commons licence and your intended use is not permitted by statutory regulation or exceeds the permitted use, you will need to obtain permission directly from the copyright holder. To view a copy of this licence, visit http://creativecommons.org/ licenses/by/4.0/. The Creative Commons Public Domain Dedication waiver (http://creativecommons.org/publicdomain/zero/1. 0/) applies to the data made available in this article, unless otherwise stated in a credit line to the data. 


\section{Background}

The Global Health Observatory data from the World Health Organization (WHO) had highlighted that in 2014, 45\% of the 147 responding countries had methadone therapy chiefly for OUD detoxification purposes [28]. The World Drug Report 2020 from the United Nations Office on Drugs and Crime (UNODC) estimated that 57.8 million people had used opioids in 2018, and $80 \%$ have lost 42 million years of "healthy life" to OUD [26]. Drug dependence has been described as a multi-factorial health disorder with a high possibility for relapse even after seeking treatment. Thus, it is imperative that people who use opioids be enrolled in opioid substitution treatment programs to cut the vicious cycle of addiction and halt the spread of infectious diseases such as HIV.

Myanmar, a country that accounted for $7 \%$ of the world's opium production [26], continued to face significant problems with opioid misuse. To curb this menace, punitive laws have been enforced, where people who use drugs can be detained and jailed for misusing illicit substances. Given the opiate misuse threat and its link with HIV spread, the Methadone Maintenance Treatment (MMT) program was first introduced in Myanmar in 2006 as a harm reduction intervention. Owing to its success, the MMT program has been expanded throughout Myanmar in line with the recommendations from the National Drug Control Policy [3]. The DDTRU first started the MMT program in Myanmar with 260 clients before it was gradually upscaled [6]. As of 2020, 26,016 people who inject drugs (PWIDs) (i.e. $28 \%$ of 93,000 estimated PWIDs in Myanmar) were taking methadone [21]. Like any other program, this harm reduction intervention could have faced challenges, obstacles and limitations in its service delivery. It would be beneficial to monitor such impediments by assessing client satisfaction towards the MMT program.

It has been pharmacologically shown that the MMT program is effective as it is associated with decreased opioid use [29]. Improvement in the quality of life (QoL) has been reported for clients enrolled in the MMT program for the short term [8], as well as long-term duration [4]. Furthermore, the MMT program clients were less likely to resort to illicit opioid use [30] and were reported to be satisfied with ancillary treatment services often provided in the MMT program [9]. Given the rapid expansion of the MMT program, several validated tools have been developed to assess clients' treatment satisfaction with the MMT program. Commonly used tools include the Client Satisfaction Questionnaire (CSQ-8) [11], the Service Satisfaction Scale (SSS-30) [19] and the Verona Service Satisfaction Scale (VSSS-32) [18]. Satisfaction with HIV/AIDS Treatment Interview Scale (SATIS) is a tool that could be modified and used to assess satisfaction towards the methadone services.
A recent situational analysis indicated that MMT programs are bound to have several limitations or barriers. They are inconvenient operational hours, long travel distance to MMT sites, lengthy registration process, coping with the continuous drug use issue among clients, long induction period, and a lack of confidentiality [14]. In view of ongoing MMT program expansion in Myanmar, it has become crucial to explore clients' satisfaction towards the MMT program and its ancillary services so that timely remedial measures could be taken to enhance the user-friendliness of the program. As such, this study aims to evaluate the satisfaction of the clients enrolled in Methadone Maintenance Treatment Program in Myanmar with the following objectives:

1) to explore clients' overall satisfaction towards the program in general;

2) to assess clients' satisfaction towards a) professional skills of the clinic staff; b) basic drug intervention (Methadone administration); c) specific intervention such as individual rehabilitation and psychotherapy,

3) to find out if any of clients' characteristics are associated with their satisfactory response.

\section{Materials and methods \\ Study design, respondents and location}

Two hundred and ten clients enrolled in the MMT program across different geographical provinces in Myanmar were recruited for this study. We used the stratified random sampling approach to recruit a quota sample of 42 respondents from each of the following locations across Myanmar; Yangon Region, Mandalay Region, Lashio (from Shan State), Kawlin (from Sagaing Region), and Mohnyin (from Kachin State). Surveys were conducted through face-to-face interviews by trained researchers at the targeted MMT facilities. Respondents were assured that this study would not collect their personally identifiable information and all the study data would be kept confidential. Each interview session lasted 25 to $30 \mathrm{~min}$. All the respondents gave informed consent and were compensated for their time.

\section{Inclusion and exclusion criteria}

The inclusion criteria for the study were as follows; a) Age 18 years and above, b) self-reported as a methadone client currently enrolled in a formal MMT program in Myanmar, and c) at least a minimum of six-month methadone treatment history. We believe six-month treatment history would be long enough to provide us with a better picture of the client's satisfaction with the MMT program and ancillary services.

Those observed to be in an incoherent state of mind and those who were reluctant to participate voluntarily were excluded from the study. 
There were full cooperation and participation from the methadone clients who were selected for participation in the survey. Drop-in-centres managers and methadone clinic doctors explained to the client the initial information session before the survey. During the interview visit, the research team also explained the importance of this survey and the potential benefit of the survey results in the operation of the methadone programme in different areas.

\section{Measures}

We collected the study data from May 2017 to July 2017. A semi-structured questionnaire was used to elicit respondents' socio-demographic characteristics such as current age, gender, employment status, MMT treatment history, HIV status, illicit drug use history, and service satisfaction with the MMT program. We also used the Verona Service Satisfaction Scale for Methadone Treatment (VSSS-MT) [5], the Addiction Severity Index- Lite (ASI) scale [13], and Quality of Life Scale developed by the World Health Organization (WHOQOLBREF) [27]. This study also used The Timeline Follow Back (TLFB survey) [15] tool to determine respondents' substance use history in the last 7 days. The responses were coded with a Likert five-point scale (1 denotes the worst satisfaction and 5 vice-versa). We reported satisfaction with methadone service (VSSS-MT score) based on the 27 VSSS-MT questions. Those rated above the 3 out of 5 Likert scales of each questionnaire were summed for the total satisfaction score. Over 80 and above was regarded as "satisfied", and the rest were "not satisfied" on that dichotomous output of satisfaction. The satisfactory responses were grouped into 3 categories: 1) professional skills of clinic staff (doctor, nurse, counsellor; 2) basic drug intervention (e.g., instruction between visits, sideeffects), and 3) specific intervention (e.g., individual rehabilitation and psychotherapy, group therapy). Two trained bilingual translators translated the survey questionnaires and validated scales from English to Myanmar (Burmese).

\section{Urinalysis}

All the respondents were required to undergo a supervised urine drug test for the following substances; Methadone, morphine, cannabis, methamphetamine, amphetamine, and benzodiazepine. All the urine specimens were appropriately discarded at the end of the interviews to avoid legal consequences.

\section{Statistical analysis}

We analysed all the study data with Stata 14.0 software. First, to identify the association between VSSS-MT Treatment satisfaction and categorical variables of patient characteristics, a Chi-squared test was performed for two tailed-test at a significance level of $p<0.05$. Next, we compared the mean scores of certain parameters (VSSS-MT scores) of interest with a $t$-test. We ran the Binary logistic regression for identifying the predictors to the outcome
"VSSS-MT score" at $p<0.05$ with confounding variables controlled. The Cox regression model for HIV infection was used to explore the relationship between treatment satisfaction and HIV status.

\section{Ethical measures}

The study had been approved by the Human Ethics and Research Committee of Universiti Sains Malaysia and then, Myanmar Ministry of Health and Sports, Department of Medical Research (No: Ethics/DMR/2017/057) [17].

\section{Results \\ Demographic characteristics}

In this study, the majority $(98.6 \%)$ of clients $(n=210)$ were males and only $1.4 \%$ were females. The mean age was 33.3 years ( $\mathrm{SD}=8.85$, range: $20-76$ years). All clients took their daily methadone supply from MMT clinics, and $83 \%$ ( $n=173 / 210$ ) were in their first-time treatment of MMT. The average daily methadone dose was $83 \mathrm{mg}(\mathrm{SD}=53$, range: $20 \mathrm{mg}-300 \mathrm{mg} /$ day), and the average duration was 28 months ( $\mathrm{SD}=28.5$, range: $6-127$ months). Almost two thirds $(63 \%, n=132)$ received $\leq 80 \mathrm{mg} /$ day dosage, while 76 (37\%) received a higher dosage of more than $80 \mathrm{mg} /$ day. The average Body Mass Index (BMI) was 20.5 (SD = 3.4, range: $14.0-33.3$ ). Regarding education status, $75.6 \%$, $(n=159 / 210)$ had primary to high school education, $21.43 \%$ had a college education, and only six clients lacked formal education. Almost half of the respondents (46.4\%) were single or divorced, while $40.58 \%$ were married.

As for the employment status in the last 3 years, most respondents $(93.43 \%)$ were employed, and only $8.57 \%$ were unemployed. Twenty-nine respondents worked as outreach workers or peer educators for people with substance use problems.

\section{Variations in treatment satisfaction rating scores}

Treatment satisfaction rating scores varied from study site to site, with scores with scores being lower in bigger cities (Yangon, Mandalay) and higher in smaller cities (Kawlin, Lashio and Mohnyin). For clients who rated 3 and above on the Likert scale of 5 for all items in the questionnaire, the mean score for satisfaction with methadone service (VSSS-MT scale) was set as 100 (range: 58-131), while the score over 80 was designated as "much satisfaction". While $88.6 \%(n=186)$ of clients were highly satisfied with their MMT program, there were variations in the ratings among the three categories: professional skill items (doctor, nurse, counsellor and worker) was rated 3.89 out of 5 , basic intervention items, 3.83 and specific intervention items 3.42. This highlights that among three categories of methadone service that captured treatment satisfaction, specific intervention category scored the lowest. 
The VSSS-MT score was mentioned based on responses to each question, as shown in Table 1.

\section{Differences in VSSS-MT scores among various characteristics of the respondents}

We also analysed whether there were significant differences in the MMT satisfaction score with different types of client behaviours and reported characteristics (See Table 2).

Among the respondents, those with a higher quality of life score on physical, psychological, social and environmental categories, those who were satisfied with their current marital and leisure status, and those who consumed alcohol had higher MMT treatment satisfaction scores of over $80(p<0.05)$ (See Fig. 1.). Those on high methadone dose $(>80 \mathrm{mg}$ /day) also had higher MMT satisfaction scores compared with clients who received the lower dose $(\leq 80 \mathrm{mg} /$ day $)(p=0.0276)$.

\section{Stepwise regression analysis to identify factors predicting the methadone service satisfaction}

To identify the predictors that were linked with the primary outcome dependent variable of MMT satisfaction, characteristics that were significantly associated with one another $(p<0.05)$ were considered in the regression analysis. The backward stepwise binary logistic regression analysis was done among the significant parameters in the model to identify final predictors to the outcome group with MMT satisfaction (See Table 3).

After adjusting the potential confounding variables, this analysis estimated the association between the independent variables and the outcome variable of the service satisfaction score category. Those who used alcohol were 6 times (aOR 5.57, 95\%CI; 1.20-25.88, $p=0.029)$, and those who had good physical health (higher score in physical quality of life) were 12 times (aOR 11.87, 95\%CI; 4.29-32.84, $p=0.000$ ) more satisfied with their MMT program. When checking for the multi collinearity, mean variance inflation factor (vif) was 1.61 and none of the variables had more than 10 . The regression model alpha ratio was set at 0.05 .

\section{Reported infection status of the patients}

About one-third $(36.5 \%, n=76)$ of respondents were on high-dose methadone (more than $80 \mathrm{mg} /$ day) and the rest $63.5 \%(n=132)$ on low-dose methadone (less than $80 \mathrm{mg} /$ day). Almost half $(47 \%, n=71 / 148)$, of the respondents reported hepatitis $\mathrm{C}$ virus infection $(\mathrm{HCV})$, $37 \%(n=74 / 200)$ reported Human Immunodeficiency Virus (HIV) infection, while $16 \%(n=34)$ reported HIV and HCV co-infection. Forty-five respondents (21.53\%) reported sexually transmitted infections, and 15 respondents (8.29\%) reported Hepatitis B infection. Out of reported HIV respondents, 68 (92\%) were on antiretroviral therapy (ART) with an average ART duration of 30 months ( $\mathrm{SD}=33$, range: $1-132$ months). Taking high methadone dose (more than $80 \mathrm{mg}$ ) was found to have a significant association with having antiretroviral therapy $(p=0.039)$ [24].

\section{Estimation of treatment satisfaction by different infection statuses}

We also ran a univariate cox proportional hazard model analysis to estimate treatment satisfaction based on the acquired data. In this survival analysis, which is similar to that of [10], "the methadone dose" was set as a "time" variable, and "Treatment satisfaction" was set as a "failure event". We then analysed the HIV status and HIV and HCV co-infection status against different methadone doses to estimate and compare incidence rates on their treatment satisfaction $(p<0.05)$.

The results show that, after controlling the methadone dose variable ( $\mathrm{IRR}=0.49, p=0.000$ ), treatment satisfaction was 2 times higher among HIV negative patients compared with HIV positive patients, and the satisfaction was 1.7 times higher among non-co-infected patients compared with co-infected patients (IRR $=0.59$, $p=0.000)$. The higher rate of satisfaction incidence among HIV negative respondents was $1.49 \%$ and among HIV positive respondents was $0.72 \%$ after adjusting the methadone dose $(\mathrm{p}=0.000)$. A higher dose was associated with increased satisfaction scores $(p=0.037)$ in HIV negative respondents [25].

Higher satisfaction incidence was $1.2 \%$ among non-coinfected patients and $0.71 \%$ among co-infected patients after adjusting for doses. In other words, co-infected patients were less likely to be satisfied with the program than non-co-infected patients $(p<0.001)$. This Cox regression, in which univariate analysis was done for the interested variable "Treatment satisfaction", and this analysis did not take into account other confounding variables. Despite the limitation of cross-sectional study type and potential bias, the estimation of the incidence rate of "treatment satisfaction" was analysed with a similar way of analysis [7].

\section{Discussion}

This study found that $88.6 \%(n=186)$ of clients were highly satisfied with the MMT program implemented in their respective localities in Myanmar.

Among three categories of methadone service that captured treatment satisfaction, the rating for professional skill items (skills of doctor, nurse, counsellor and workers) scored the highest (3.89 out of 5), while items in specific intervention category scored the lowest. A Vietnamese study [22] also reported high satisfaction in professional skill items. It was reported that MMT programs that offered ancillary services as 
Table 1 Table shows VSSS-MT Treatment satisfaction score among respondents

\begin{tabular}{|c|c|c|c|c|}
\hline Respondent characters & Sub groups & Number (n) & Total VSSS-MT score (SD) & $p$ value \\
\hline Methadone dose categories & $\begin{array}{l}\text { less than or equal } 80 \mathrm{mg} \\
\text { more than } 80 \mathrm{mg}\end{array}$ & $\begin{array}{l}132 \\
76\end{array}$ & $\begin{array}{l}98.61(13.97) \\
103.11(14.28)\end{array}$ & $0.0276^{* *}$ \\
\hline Methadone duration & $\begin{array}{l}\text { less than or equal } 2.4 \text { years } \\
\text { more than } 2.4 \text { years }\end{array}$ & $\begin{array}{l}120 \\
89\end{array}$ & $\begin{array}{l}100.83(14.40) \\
99.40(13.93)\end{array}$ & 0.4755 \\
\hline Methadone treat time & $\begin{array}{l}\text { First time treatment } \\
\text { More than first time }\end{array}$ & $\begin{array}{l}173 \\
35\end{array}$ & $\begin{array}{l}101.34(13.95) \\
94.74(14.46)\end{array}$ & $0.0120^{* *}$ \\
\hline - less than or equal $80 \mathrm{mg}$ & $\begin{array}{l}\text { First time treatment } \\
\text { More than first time }\end{array}$ & $\begin{array}{l}109 \\
22\end{array}$ & $\begin{array}{l}100.03(13.45) \\
91.54(14.96)\end{array}$ & $0.0091^{* *}$ \\
\hline - more than $80 \mathrm{mg}$ & $\begin{array}{l}\text { First time treatment } \\
\text { More than first time }\end{array}$ & $\begin{array}{l}63 \\
13\end{array}$ & $\begin{array}{l}103.71(14.67) \\
100.15(12.27)\end{array}$ & 0.4167 \\
\hline - less than or equal 2.4 years & $\begin{array}{l}\text { First time treatment } \\
\text { More than first time }\end{array}$ & $\begin{array}{l}95 \\
24\end{array}$ & $\begin{array}{l}102.68(13.52) \\
93.54(15.98)\end{array}$ & 0.0052 \\
\hline - more than 2.4 years & $\begin{array}{l}\text { First time treatment } \\
\text { More than first time }\end{array}$ & $\begin{array}{l}78 \\
11\end{array}$ & $\begin{array}{l}99.69(14.37) \\
97.36(10.61)\end{array}$ & 0.6067 \\
\hline HIV status (HIV) & $\begin{array}{l}\text { Not infected } \\
\text { Infected }\end{array}$ & $\begin{array}{l}126 \\
74\end{array}$ & $\begin{array}{l}99.23(13.84) \\
102.35(14.81)\end{array}$ & 0.1351 \\
\hline Hepatitis C status (HCV) & $\begin{array}{l}\text { Not infected } \\
\text { Infected }\end{array}$ & $\begin{array}{l}77 \\
71\end{array}$ & $\begin{array}{l}103.21(13.35) \\
98.66(14.03)\end{array}$ & $0.0452^{* *}$ \\
\hline Hepatitis B status (HBV) & $\begin{array}{l}\text { Not infected } \\
\text { Infected }\end{array}$ & $\begin{array}{l}166 \\
15\end{array}$ & $\begin{array}{l}101.14(14.04) \\
101.73(12.19)\end{array}$ & 0.8754 \\
\hline Tuberculosis (TB) treatment history & $\begin{array}{l}\text { Not treated } \\
\text { Treated }\end{array}$ & $\begin{array}{l}147 \\
54\end{array}$ & $\begin{array}{l}100.92(13.71) \\
100.65(14.00)\end{array}$ & 0.9021 \\
\hline Sexually Transmitted infection (STI) history & $\begin{array}{l}\text { Not infected } \\
\text { Infected }\end{array}$ & $\begin{array}{l}164 \\
45\end{array}$ & $\begin{array}{l}100.06(13.69) \\
100.80(16.01)\end{array}$ & 0.7577 \\
\hline Age & $\begin{array}{l}\text { Younger and equal } 35 \text { years } \\
\text { Older than } 35 \text { years }\end{array}$ & $\begin{array}{l}128 \\
81\end{array}$ & $\begin{array}{l}100.82(14.43) \\
99.27(13.82)\end{array}$ & 0.4433 \\
\hline Body Mass Index (BMI) & $\begin{array}{l}\text { Less than mean BMI (20.5) } \\
\text { More than mean BMI }\end{array}$ & $\begin{array}{l}122 \\
84\end{array}$ & $\begin{array}{l}100.33(14.87) \\
100.48(13.06)\end{array}$ & 0.9412 \\
\hline Currently on antiretroviral therapy (ART) & $\begin{array}{l}\text { No } \\
\text { On treatment }\end{array}$ & $\begin{array}{l}141 \\
68\end{array}$ & $\begin{array}{l}99.08(13.53) \\
102.59(15.29)\end{array}$ & 0.0938 \\
\hline Education & $\begin{array}{l}\text { Up to primary } \\
\text { More than primary }\end{array}$ & $\begin{array}{l}48 \\
161\end{array}$ & $\begin{array}{l}104.77(15.35) \\
98.86(13.58)\end{array}$ & $0.0110^{* *}$ \\
\hline Recent work & $\begin{array}{l}\text { Unemployed } \\
\text { Employed }\end{array}$ & $\begin{array}{l}24 \\
182\end{array}$ & $\begin{array}{l}96.95(15.41) \\
100.93(13.81)\end{array}$ & 0.1926 \\
\hline Current Peer/ Outreach & $\begin{array}{l}\text { No } \\
\text { Peer/outreach }\end{array}$ & $\begin{array}{l}180 \\
29\end{array}$ & $\begin{array}{l}100.52(14.27) \\
98.38(13.77)\end{array}$ & 0.4528 \\
\hline ASI for Employment & $\begin{array}{l}\text { Low score } \\
\text { High score }\end{array}$ & $\begin{array}{l}102 \\
104\end{array}$ & $\begin{array}{l}99.39(13.39) \\
100.83(15.06)\end{array}$ & 0.4711 \\
\hline ASI for Alcohol Use & $\begin{array}{l}\text { Low score } \\
\text { High score }\end{array}$ & $\begin{array}{l}28 \\
36\end{array}$ & $\begin{array}{l}105.96(10.96) \\
102.22(14.48)\end{array}$ & 0.2600 \\
\hline ASI for Drug Use & $\begin{array}{l}\text { Low score } \\
\text { High score }\end{array}$ & $\begin{array}{l}162 \\
47\end{array}$ & $\begin{array}{l}100.96(14.21) \\
97.66(13.97)\end{array}$ & 0.1604 \\
\hline ASI for Legal Status & $\begin{array}{l}\text { Low score } \\
\text { High score }\end{array}$ & $\begin{array}{l}14 \\
14\end{array}$ & $\begin{array}{l}101.64(15.57) \\
94.29(10.86)\end{array}$ & 0.1590 \\
\hline ASI for Family/ Social Status & $\begin{array}{l}\text { Low score } \\
\text { High score }\end{array}$ & $\begin{array}{l}139 \\
70\end{array}$ & $\begin{array}{l}100.90(13.71) \\
98.87(15.09)\end{array}$ & 0.3306 \\
\hline Marital status & $\begin{array}{l}\text { Currently married } \\
\text { Single/separated }\end{array}$ & $\begin{array}{l}84 \\
122\end{array}$ & $\begin{array}{l}98.62(14.51) \\
101.48(14.01)\end{array}$ & 0.1568 \\
\hline Income & $\begin{array}{l}\text { Lower (than average) } \\
\text { Higher }\end{array}$ & $\begin{array}{l}131 \\
77\end{array}$ & $\begin{array}{l}99.65(14.97) \\
101.18(12.89)\end{array}$ & 0.4542 \\
\hline Current marital status satisfaction & Not satisfied & 20 & $96.65(19.01)$ & 0.2375 \\
\hline
\end{tabular}


Table 1 Table shows VSSS-MT Treatment satisfaction score among respondents (Continued)

\begin{tabular}{|c|c|c|c|c|}
\hline Respondent characters & Sub groups & Number (n) & Total VSSS-MT score (SD) & $p$ value \\
\hline & Satisfied & 189 & $100.60(13.59)$ & \\
\hline WHO Quality of life (QOL) total score & $\begin{array}{l}\text { Low } \\
\text { High }\end{array}$ & $\begin{array}{l}88 \\
121\end{array}$ & $\begin{array}{l}93.63(14.42) \\
105.02(11.96)\end{array}$ & $0.0001^{* * *}$ \\
\hline Physical QOL score & $\begin{array}{l}\text { Low } \\
\text { High }\end{array}$ & $\begin{array}{l}53 \\
156\end{array}$ & $\begin{array}{l}88.94(15.06) \\
104.05(11.65)\end{array}$ & $0.0001^{* * *}$ \\
\hline Psychological QOL score & $\begin{array}{l}\text { Low } \\
\text { High }\end{array}$ & $\begin{array}{l}41 \\
168\end{array}$ & $\begin{array}{l}91.07(15.32) \\
102.45(12.99)\end{array}$ & $0.0001^{* * *}$ \\
\hline Social QOL score & $\begin{array}{l}\text { Low } \\
\text { High }\end{array}$ & $\begin{array}{l}71 \\
138\end{array}$ & $\begin{array}{l}94.86(15.47) \\
102.98(12.68)\end{array}$ & $0.0001^{* * *}$ \\
\hline Environmental QOL score & $\begin{array}{l}\text { Low } \\
\text { High }\end{array}$ & $\begin{array}{l}54 \\
155\end{array}$ & $\begin{array}{l}90.81(14.83) \\
103.50(12.42)\end{array}$ & $0.0001^{* * *}$ \\
\hline Current leisure status satisfaction & $\begin{array}{l}\text { Not satisfied } \\
\text { Satisfied }\end{array}$ & $\begin{array}{l}29 \\
180\end{array}$ & $\begin{array}{l}92.38(16.59) \\
101.48(13.39)\end{array}$ & $0.0012^{* *}$ \\
\hline - Current leisure status with family & $\begin{array}{l}\text { Not satisfied } \\
\text { Satisfied }\end{array}$ & $\begin{array}{l}119 \\
90\end{array}$ & $\begin{array}{l}100.03(14.27) \\
100.48(14.15)\end{array}$ & 0.8200 \\
\hline - Current leisure status with friend & $\begin{array}{l}\text { Not satisfied } \\
\text { Satisfied }\end{array}$ & $\begin{array}{l}135 \\
74\end{array}$ & $\begin{array}{l}99.52(14.76) \\
101.50(13.08)\end{array}$ & 0.3354 \\
\hline - Current leisure status alone & $\begin{array}{l}\text { Not satisfied } \\
\text { Satisfied }\end{array}$ & $\begin{array}{l}159 \\
50\end{array}$ & $\begin{array}{l}101.09(13.71) \\
97.44(15.42)\end{array}$ & 0.1123 \\
\hline \multicolumn{5}{|l|}{ Abuse encountered within 30 days } \\
\hline - Psychological abuse & $\begin{array}{l}\text { Not experienced } \\
\text { Experienced }\end{array}$ & $\begin{array}{l}183 \\
22\end{array}$ & $\begin{array}{l}100.01(13.91) \\
101.05(16.64)\end{array}$ & 0.7474 \\
\hline - Physical abuse & $\begin{array}{l}\text { Not experienced } \\
\text { Experienced }\end{array}$ & $\begin{array}{l}203 \\
1\end{array}$ & $\begin{array}{l}100.10(14.26) \\
102.00(.)\end{array}$ & . \\
\hline - Sexual abuse & $\begin{array}{l}\text { Not experienced } \\
\text { Experienced }\end{array}$ & $\begin{array}{l}204 \\
1\end{array}$ & $\begin{array}{l}100.07(14.20) \\
111.00(.)\end{array}$ & \\
\hline \multicolumn{5}{|l|}{ Urine illicit substance findings } \\
\hline - Urine Morphine & $\begin{array}{l}\text { Absent } \\
\text { Present }\end{array}$ & $\begin{array}{l}93 \\
116\end{array}$ & $\begin{array}{l}100.62(14.13) \\
99.90(14.29)\end{array}$ & 0.7137 \\
\hline - Urine Tetrahydrocannabinol (THC) & $\begin{array}{l}\text { Absent } \\
\text { Present }\end{array}$ & $\begin{array}{l}185 \\
24\end{array}$ & $\begin{array}{l}100.42(14.40) \\
98.71(12.59)\end{array}$ & 0.5802 \\
\hline - Urine Methamphetamine & $\begin{array}{l}\text { Absent } \\
\text { Present }\end{array}$ & $\begin{array}{l}158 \\
51\end{array}$ & $\begin{array}{l}100.93(14.62) \\
98.02(12.65)\end{array}$ & 0.2034 \\
\hline - Urine Amphetamine & $\begin{array}{l}\text { Absent } \\
\text { Present }\end{array}$ & $\begin{array}{l}191 \\
18\end{array}$ & $\begin{array}{l}100.19(14.50) \\
100.56(10.55)\end{array}$ & 0.9167 \\
\hline - Urine Benzodiazepine & $\begin{array}{l}\text { Absent } \\
\text { Present }\end{array}$ & $\begin{array}{l}137 \\
72\end{array}$ & $\begin{array}{l}100.84(14.95) \\
99.04(12.62)\end{array}$ & 0.3853 \\
\hline Last heroin injection within 30 days & $\begin{array}{l}\text { No } \\
\text { Yes }\end{array}$ & $\begin{array}{l}93 \\
115\end{array}$ & $\begin{array}{l}102.35(14.15) \\
98.44(14.08)\end{array}$ & $0.0483^{* *}$ \\
\hline Frequency of injection & $\begin{array}{l}\text { No or few injections (mean = 7) } \\
\text { Higher }\end{array}$ & $\begin{array}{l}96 \\
112\end{array}$ & $\begin{array}{l}102.46(13.64) \\
98.35(14.48)\end{array}$ & $0.0373^{* *}$ \\
\hline Needle sharing within 30 days & $\begin{array}{l}\text { Not shared } \\
\text { Shared }\end{array}$ & $\begin{array}{l}99 \\
4\end{array}$ & $\begin{array}{l}99.21(15.26) \\
102.00(1.41)\end{array}$ & 0.7169 \\
\hline Life time sharing of needle and syringes & $\begin{array}{l}\text { Not shared } \\
\text { Shared }\end{array}$ & $\begin{array}{l}106 \\
102\end{array}$ & $\begin{array}{l}98.72(12.66) \\
101.58(15.47)\end{array}$ & 0.1452 \\
\hline
\end{tabular}

Independent t-test $p$ value: ** significance $<0.05,{ }^{* * *}$ significance $<0.001$

Table explanation: This table explains the result of the significant difference of the total score of the VSSS-MT questionnaire with the respondent characteristics

specific intervention improved MMT satisfaction ([12, 22]. It was reported that limitations or barriers such as dosing spaces, waiting areas and staff shortage could affect treatment satisfaction [2]. Thus the specific intervention component in MMT programs could be enhanced by providing ancillary services such as counselling, medical services (Individual rehabilitation), psycho-social services (psychotherapy and group therapy), and psychiatric care. Thus, the specific intervention component in MMT programs 
Table 2 Table shows total VSSS-MT score differences by the characteristics of patients

\begin{tabular}{lll}
\hline Variable & $\begin{array}{l}\text { Adjusted Odds Ratio (aOR) } \\
(\mathbf{9 5 \%} \mathbf{C l})\end{array}$ & $\boldsymbol{p}$ value \\
\hline Alcohol & $5.57(1.2,25.88)$ & $0.029^{* *}$ \\
$\begin{array}{l}\text { Physical quality of life }(\mathrm{QOL}) \\
\text { score }\end{array}$ & $11.87(4.29,32.84)$ & $0.000^{* * *}$ \\
\hline
\end{tabular}

Stepwise binary logistic regression, $p$ value: ${ }^{* *}$ significance $p<0.05$, ***significance $p<0.001$.

could be enhanced by providing ancillary services such as counselling, medical services (Individual rehabilitation), psycho-social services (psychotherapy and group therapy), and psychiatric care. As the specific intervention focuses on individual and group counselling, family support, and the centre supports at home, the methadone intervention can be more effective if integrated with individual and/or group counselling, employment or family services [1]. Moving in this direction could improve clients' treatment adherence and satisfaction.

Regarding the program categories, the ratings for satisfaction in this study were higher than those reported by a study from Spain (3.83 vs 3.5 for basic intervention items and 3.42 vs 3.1 for specific intervention) [23]. People in Myanmar are known for their friendliness, easy-going lifestyle and a high tolerance for life's inconveniences. This might be a factor for the high satisfaction scores observed in this study.
Many factors influence MMT treatment satisfaction. These include recent heroin injections, use of benzodiazepines, HCV infection, high addiction severity index (ASI), alcohol consumption and family/ social status. Limitations or barriers in methadone treatment such as dosing spaces, waiting areas and staff shortage [2], methadone dispensing hour, methadone dose change, the number of patients per centre, frequency of information about methadone dose changes, and lower social dysfunction subscale of General Health Questionnaire28 [23]; could also affect treatment satisfaction.

Our stepwise binary logistic regression result showed alcohol consumption and physical health were significantly associated with methadone treatment satisfaction. Although Myanmar is a majority Buddhist country, alcohol consumption is common among its people.

There were a few limitations in this study. First, respondents for this study were randomly recruited from a few MMT clinics. Hence there could be bias by not enrolling clients unsatisfied with their MMT program. There is a limitation to make generalisations from the results bias the data were based on one-time selfreporting. The result's further potential bias could be contributed by the selection criteria of a minimum 6month treatment. The National indicator from the DDTRU (Drug Dependency Treatment and Research Unit) specified the client retention in the methadone treatment as "those clients who were taking methadone at least 6-month period", and this survey excluded the satisfactory situation of the clients who were less than 6

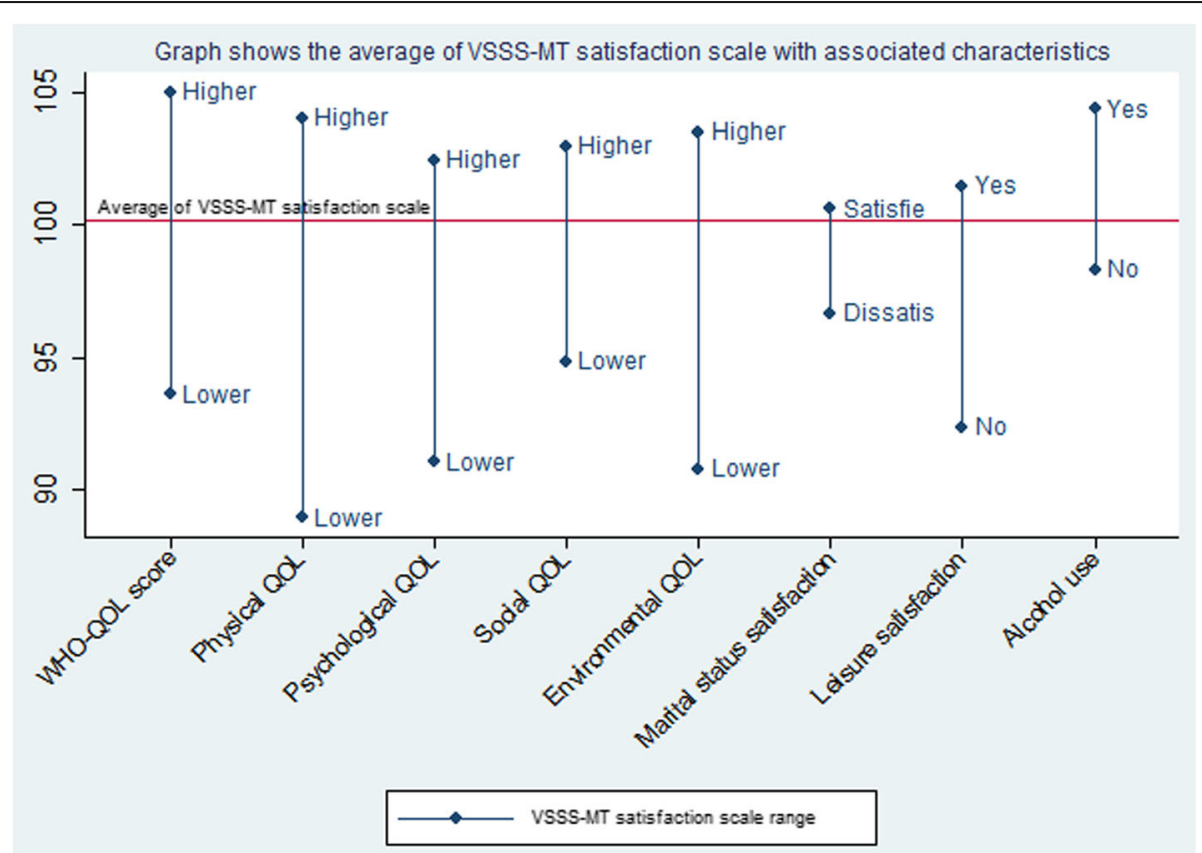

Fig. 1 Graph shows the average of VSSS-MT satisfaction score (scale) with associated characteristics. Legend: Y-axis shows the average result of the VSSS-MT total score 
Table 3 Table shows correlates of VSSS-MT score from stepwise

\begin{tabular}{|c|c|c|c|}
\hline Variable & $\begin{array}{l}\text { Frequency } \\
\text { (n and \%) }\end{array}$ & $\begin{array}{l}\text { Mean (SD) VSSS-MT } \\
\text { score }^{\mathrm{a}}\end{array}$ & Range/ Categories \\
\hline $\begin{array}{l}\text { Verona Service Satisfaction Scale for Methadone-Treatment (VSSS-MT) score } \\
\text { (total) }\end{array}$ & 209 (100\%) & $100.22(14.19)$ & $58-131$ \\
\hline Not much satisfied & $23(11 \%)$ & & \\
\hline Much satisfied & $186(89 \%)$ & & \\
\hline \multicolumn{4}{|l|}{ VSSS Item Categories } \\
\hline Professional Skills Items & 209 & $3.89(0.64)$ & $1.5-5$ \\
\hline Basic Interventions Items & 209 & $3.83(0.54)$ & $2.3-5$ \\
\hline Specific Interventions Items & 209 & $3.42(0.68)$ & $1.5-4.9$ \\
\hline \multicolumn{4}{|l|}{ VSSS Items Description } \\
\hline 1. Helping patient deal with problem & 209 & $3.77(0.96)$ & Basic skill items \\
\hline 2. Doctors' ability to listen & 208 & $3.97(0.83)$ & Professional Skills Items \\
\hline 3. Psychologists' ability to listen & 206 & $3.99(0.86)$ & Professional Skills Items \\
\hline 4. Doctors' manner & 208 & $3.92(0.84)$ & Professional Skills Items \\
\hline 5. Psychologists' manner & 204 & $4.02(0.77)$ & Professional Skills Items \\
\hline 6. Referring to other specialists & 201 & $3.76(0.87)$ & Basic skill items \\
\hline 7. Overall satisfaction & 208 & $4.22(0.76)$ & Basic skill items \\
\hline 8. Nurses' manner & 209 & $3.91(0.84)$ & Professional Skills Items \\
\hline 9. Social workers' manner & 205 & $3.73(1.00)$ & Professional Skills Items \\
\hline 10. Improving relationship between patient and relatives & 208 & $4.00(0.84)$ & Basic skill items \\
\hline 11. Helping family members to understand patient's problems & 209 & $4.01(0.81)$ & Basic skill items \\
\hline 12. Nurses' knowledge of patient's medical history & 209 & $3.78(0.94)$ & Basic skill items \\
\hline 13. Information on addiction & 209 & $3.72(0.93)$ & Basic skill items \\
\hline 14. Helping patient in relationships outside the family & 209 & $3.44(1.04)$ & Basic skill items \\
\hline 15. Instructions between visits & 209 & $3.79(0.85)$ & Basic skill items \\
\hline 16. Helping patient to look after himself & 209 & $3.98(0.77)$ & Basic skill items \\
\hline 17. Nurses' ability to listen & 208 & $3.83(0.84)$ & Professional Skills Items \\
\hline 18. Social workers' ability to listen & 209 & $3.75(0.92)$ & Professional Skills Items \\
\hline 19. Help received for methadone side effects & 207 & $3.88(0.85)$ & Basic skill items \\
\hline 20. Individual rehabilitation & 209 & $3.64(1.07)$ & $\begin{array}{l}\text { Specific Interventions } \\
\text { Items }\end{array}$ \\
\hline 21. Individual psychotherapy & 209 & $3.67(1.05)$ & $\begin{array}{l}\text { Specific Interventions } \\
\text { Items }\end{array}$ \\
\hline 22. Family therapy & 208 & $4.19(0.81)$ & $\begin{array}{l}\text { Specific Interventions } \\
\text { Items }\end{array}$ \\
\hline 23. Activities organised by centre & 209 & $3.82(0.97)$ & $\begin{array}{l}\text { Specific Interventions } \\
\text { Items }\end{array}$ \\
\hline 24. Group psychotherapy & 209 & $3.33(1.06)$ & $\begin{array}{l}\text { Specific Interventions } \\
\text { Items }\end{array}$ \\
\hline 25. Sheltered work & 208 & $3.03(1.23)$ & $\begin{array}{l}\text { Specific Interventions } \\
\text { Items }\end{array}$ \\
\hline 26. Help by the centre at home & 204 & $2.51(1.27)$ & $\begin{array}{l}\text { Specific Interventions } \\
\text { Items }\end{array}$ \\
\hline 27. Help to join in activities separate from the centre & 209 & $3.16(1.17)$ & $\begin{array}{l}\text { Specific Interventions } \\
\text { Items }\end{array}$ \\
\hline
\end{tabular}

${ }^{\mathrm{a}}$ Table explanation: This table explains the result of the mean score for each components of the VSSS-MT questionnaire set 
months of the treatment. The 2018 annual data [16] showed $70 \%$ received at least 6 months, and we could interpret that the survey results were derived from the samples representing the majority of the clients. As the respondents were patients currently taking methadone, they might be reluctant to take risks in expressing negative views; Hawthorn's effect thus could not be ruled out despite our efforts in ensuring confidentiality. We analysed this study with the Chi-squared test repeatedly to identify the satisfaction of the methadone clients. So, there could be a potential increase in type I error as there were multiple comparisons to test the null hypotheses [20]. Finally, factors like early drop-outs and refusal to participate in the study, could be a threat to the internal validity and generalizability of the results. In conclusion, our results indicated that clients in the MMT program in Myanmar were highly satisfied with their methadone treatment program. However, the lower percentage of satisfied clients for the specific intervention category compared with staff and basic drug management categories highlights the need for improvement in the Specific intervention component in MMT programs by providing ancillary services such as counselling, medical services (Individual rehabilitation), psycho-social services (psychotherapy, and group therapy), and psychiatric care.

\section{Abbreviations \\ aOR: Adjusted Odds Ratio; ART: Antiretroviral therapy; ASI: Addiction Severity Index; BMI: Body Mass Index; DDTRU: Drug Dependency Treatment and Research Unit; HCV: Hepatitis C Virus; HIV: Human Immunodeficiency Virus; IRR: Incidence Rate Ratio; MMT: Methadone Maintenance Treatment; PWID: People Who Inject Drugs; SD: Standard Deviation; VSSS-MT: The Verona Service Satisfaction Scale for Methadone-Treatment; WHO: World Health Organization; WHOQOL-BREF: generic Quality of Life Scale developed through the World Health Organization}

\begin{abstract}
Acknowledgements
I sincerely appreciate my academic mentor, Prof Nyunt Wai, for his critical review and editing of this manuscript revision and guidance throughout my academic and professional works. The authors would like to thank all survey respondents; specifically to Dr. Nanda Myo Aung Wan, Drug Dependency Treatment and Research Unit (DDTRU) Programme Manager in Myanmar who supported proposal development. Further appreciation goes to Dr. Ohnmar Thaung, U Thet Swe, Dr. Phyo Myat, Dr. Nay Lin, Dr. Myo Minn Minn and harm reduction organizations in Myanmar (Myanmar Anti-Narcotic Association, Asian Harm Reduction Network, Burnet Institute) for supporting survey data collection.
\end{abstract}

\section{Authors' contributions}

Sun Tun and Nyunt Wai contributed to designing, implementing, analysing, and critical reviewing and writing the manuscript. B. Vicknasingam and Darshan Singh contributed to this manuscript's designing and analysis. The authors read and approved the final manuscript

\section{Funding}

The authors declared that the research and publication of their article were self-funded for academic purposes.

\section{Availability of data and materials}

The [.dta] data used of the findings in this study are available from the Centre for Drug Research upon the approval.

\section{Declarations}

\section{Ethical approval and consent to participate}

This research approval was granted from the Human Ethics Committee of the Universiti Sains Malaysia (No:USM/ JEPeM/16080269) (University of Science, Malaysia) and Department of Medical Research (No: Ethics/DMR/ 2017/057), and then, Ministry of Health and Sports, [17].

\section{Consent for publication}

All authors read and approved the final manuscript submission. All authors agreed to publish this prepared manuscript and no other submission of this manuscript to another journal concurrently.

\section{Competing interests}

All authors declare that there is no competing interest.

Accepted: 17 December 2021

Published online: 03 January 2022

\section{References}

1. Anon., 2018. Opioid Addiction. In: Principles of Drug Addiction Treatment: A Research-Based Guide (Third Edition). s.l.:s.n.

2. Aziz, Z. \& Chong, N. J., 2015. A Satisfaction Survey of Opioid-Dependent Patients with Methadone Maintenance Treatment. J Substance Abuse TreatmentSedere, Volume 53, pp. 47-51.

3. CCDAC, 2018. Myanmar Drug Control Policy, s.l.: Central Committee for Drug Abuse Control

4. Chou Y-C, et al. Improvement of quality of life in methadone treatment patients in northern Taiwan: a follow-up study. BMC Psychiatry. 2013; 13(190).

5. Cobos, J. P. d. I. et al., 2002. Development and psychometric properties of the Verona Service Satisfaction Scale for methadone-treated opioiddependent patients (VSSS-MT). Drug Alcohol Dependence, 68(2), pp. 209214.

6. DDTRU. National Drug Abuse Prevention and Control Programme. Yangon: DDTRU; 2015.

7. DeMonte JB, et al. Rapid report on estimating incidence from crosssectional data. Ann Epidemiol. 2021:106-108.e1.

8. Fei JTB, Yee A, Habil MHB, Danaee M. Effectiveness of Methadone Maintenance Therapy and Improvement in Quality of Life Following a Decade of Implementation. J Substance Abuse Treatment. 2016;13 July: 50-6.

9. Ghani MA, et al. An exploratory qualitative assessment of self-reported treatment outcomes and satisfaction among patients accessing an innovative voluntary drug treatment centre in Malaysia. Int J Drug Policy. 2015:26(2):175-82

10. Hansen NB, Lambert MJ. An Evaluation of the Dose-Response Relationship in Naturalistic Treatment Settings Using Survival Analysis. Mental Health Services Research. 2003;5(1).

11. Larsen DL, Attkisson CC, Hargreaves WA, Nguyen TD. Assessment of client/ patient satisfaction: Development of a general scale. Evaluation and Program Planning. 1979;2(3):197-207.

12. Li L, et al. Factors related to client satisfaction with methadone maintenance treatment in China. J Substance Abuse Treatment. 2017;77: 201-6.

13. McLellan, T., Cacciola, J., Carise, D. \& Coyne, T. H., 1999. Addiction Severity Index Lite - CF, s.l.: s.n

14. Myanmar U. Situational Analysis on Drug Use, HIV and the Response in Myanmar: Looking Forward. Yangon: UNAIDS; 2015.

15. NIDA-CTN, 2014. Instrument: Timeline Followback Method Assessment. [Online] Available at: https://cde.drugabuse.gov/sites/nida_cde/files/ TimeLineFollowBack_2014Mar24.pdf

16. Program, N. A., 2020. Progress Report 2018, s.l.: s.n.

17. Research, D. o. M., 2017. http://www.ercdmrlm.org. [Online] Available at: http://www.ercdmrlm.org/pdf/2017_Certificate_List.pdf

18. Rugger M, Rosa D, Bisoffi G, Greenfield T. Factor analysis of the Verona Service Satisfaction Scale-82 and development of reduced version. Int J Methods in Psychiatric Res. 1996;6(1):23-38.

19. Sederer LL, Dickey B. The Client Satisfaction Questionnaire (CSQ) Scales. In: Outcome Assessment in Clinical Practice. s.l.:Baltimore. MD: Williams \& Wilkins; 1996. 
20. stat.berkeley.edu, n.d Multiple Hypothesis Testing and False Discovery Rate. [Online] Available at: https://www.stat.berkeley.edu/hhuang/STAT141/ Lecture-FDR.pdf [Accessed 6 Nov 2021]

21. The Global Fund Programme Myanmar, 2020. The Global Fund Programme in Myanmar. [Online] Available at: https://pr-myanmar.org/en/disease/hiv [Accessed 13 Aug 2021].

22. Tran BX, Nguyen LH, Phan HTT, Latkin CA. Patient Satisfaction with Methadone Maintenance Treatment in Vietnam: A Comparison of Different Integrative-Service Delivery Models. PLOS One. 2015;10(11):e0142644.

23. Trujols J, et al. Patient satisfaction with methadone maintenance treatment: The relevance of participation in treatment and social functioning. Drug and Alcohol Dependence. 2012;123:41-7.

24. Tun S, Vicknasingam B, Singh D. Higher methadone dose reduces risky drug injecting behaviours among methadone clients in Myanmar. Emerg Trends Drugs Addictions Health. 2021a, 2021;1.

25. Tun, S., Vicknasingam, B. \& Singh, D., 2021 b. Methadone dose affects service satisfaction of HIV infected PWIDs treated on methadone in Myanmar. Int J Epidemiol, 50, (Supplement_1), p. dyab168.669.

26. UNODC. World Drug Report 2020. s.l.: UNODC; 2020.

27. WHO, 2004. The World Health Organization Quality of Life (WHOQOL)-BREF, s.l.: s.n.

28. WHO, 2014. Pharmacotherapy with methadone, s.l. s.n.

29. Yl H, et al. Long-term outcomes after randomization to buprenorphine/ naloxone versus methadone in a multi-site trial. Addiction. 2016;111(4):695705.

30. Zhang $\mathrm{L}$, et al. Methadone Maintenance Treatment Participant Retention and Behavioural Effectiveness in China: A Systematic Review and MetaAnalysis. PLOSone. 2013.

\section{Publisher's Note}

Springer Nature remains neutral with regard to jurisdictional claims in published maps and institutional affiliations.

Ready to submit your research? Choose BMC and benefit from:

- fast, convenient online submission

- thorough peer review by experienced researchers in your field

- rapid publication on acceptance

- support for research data, including large and complex data types

- gold Open Access which fosters wider collaboration and increased citations

- maximum visibility for your research: over $100 \mathrm{M}$ website views per year

At $\mathrm{BMC}$, research is always in progress.

Learn more biomedcentral.com/submissions 